\title{
Der preussische Medicinaletat für das Jahr 1900.
}

Danernde Ausgaben. Unter den Universitaiten ist bei der Universität Königsberg eine Summe von $2750 \mathrm{M}$. eingestellt zur Umwandlung eines Extraordinariats für Hygiene in ein Ordinariat: damit sind alle preussischen Universitäten mit ordentlichen Professuren für Hygiene ausgestattet. An der Frauenklinik wird eine weitere Assistentenstelle neu eingerichtet, der Ausgabefond der Augenklinik wird um $1200 \mathrm{M}$. vermehrt. - In Berlin wird ein Ersatzordinariat (künftig wegfallend) mit $5400 \mathrm{M}$., ein Extraordinariat für Untersuchungen mit Röntgenstrahlen, im physiologischen Institut eine Assistentenstelle neu eingerichtet. Erhöht werden die sächlichen Ausgabefonds des anatomischen Instituts, des physiologischen Instituts, der medicinischen Poliklinik, der medicinischen Kliniken, der chirurgischen Poliklinik der Charité, der Kliniken für Hals- und Nasen-, für Ohren- und für Augenkrankheiten der Charité, der Nervenklinik, der Poliklinik für Haut- und Geschlechtskrankheiten. Zur Begründung eines Röntgen-Instituts sind 11500 M. (Gehalt eines Assistenten etc.) eingestellt. - Für Greifs wald wird ein Ersatzordinariat (künftig wegfallend) geschaffen, im anatomischen Institut ein Prosektor (künftig wegfallend), in der Frauenund Augenklinik ein weiterer Assistent angestellt, die sächlichen Ausgabefonds derselben beiden Kliniken erhöht. - In Breslau wird am physiologischen Institut, an der medicinischen Klinik, an der Klinik und Poliklinik für Kinderkrankheiten je eine neue Assistentenstelle geschaffen und der Ausgabefond des anatomischen Instituts, der klinischen Anstalten, der Klinik für Kinderkrankheiten erhöht. - In $\mathrm{H}$ alle wird an der chirurgischen Klinilk eine Assistentenstelle in eine Oberarztstelle umgewandelt. - In Kiel soll ein Ordinariat für Psychiatrie errichtet werden, im pathologischen Institut eine Assistentenstelle in eine Prosectur umgewandelt, an mehreren Kliniken Freistellen für arme Kranke geschaffen werden. Der Ausgabefond der chirurgischen Klinik wird verstärkt, und die Poliklinik für Ohren-, Hals- und Nasenkrankkeiten erhält eine Subvention. - Für Göttingen ist nur eine Erhöhung des Ausgabefonds beim anatomischen Institut in Aussicht genommen, ebenso für Marburg eine solche der chirurgischen und Augen-Klinik. - In Bonn wird die Remuneration einiger Assistentenstellen auf die übliche Höhe gebracht, die Subvention der Poliklinik für Ohrenkrankheiten gesteigert.

In dem Abschnitt Medicinalwesen sind unter der Rubrik „Besoldungen" keine bemerkenswerthen Veränderungen vorhanden. Bei dem Titel "Sachliche Ausgaben" ist beiläufig zu erwähnen, dass etwaige Ersparnisse an dem zur Remunerirung für Staatsprüfungen verwandten Fond zur Unterstützung bedürftiger Studirenden der Medicin und Pharmacie, sowie bedürftiger Aerzte und deren Hinterbliebenen verwandt werden können. - Bei den Unterrichts-, Heilund Wohlthätigkeitsanstalten " ist die Uebernahme der Krankenabtheilung des Instituts für Infectionskrankheiten auf die Charité bemerkenswerth. Neu eingestellt wird ein Assistent an der Station zur Heilung und Erforschung der Wuthkrankheit; an derselben Station werden Mittel zu wissenschaftlichen Untersuchungen und zu freien Verpflegungstagen an unbemittelte Kranke, insgesammt 7000 M., ausgeworfen. - Für das Institut für experimentelle Therapie zu Frankfurt a. M. sind $65000 \mathrm{M}$. an Ausgaben eingestellt; diesem stehen zu erwartende Einnahmen aus der Prüfung von Serumpräparaten und anderen Heilmitteln etc. in Höhe von $48000 \mathrm{M}$. entgegen. Für das hygienische Institut in Posen sind $28622 \mathrm{M}$. aufgewandt. Zur Vermehrung des hilfsärztlichen Personals sind ferner $6000 \mathrm{M}$. künftig wegfallend - eingestellt. - Unter den „Sonstigen Ausgaben für medicinal-polizeiliche Zwecke" figuriren 5000 M. zur Unterstiutzung für active Medicinalbeamte und $60000 \mathrm{M}$. zu Unterstützungen für ausgeschiedene Medicinalbeamte, sowie für Wittwen und Waisen von Medicinalbeamten.

Für das gesammte Medicinalwesen sind an jährlichen dauernden Ausgaben 1976059 M. angesetzt.

Einmalige und ausserordentliche Ausgaben. Universitäten. In Königsberg ist für den Neubau des hygienischen Instituts die letzte Rate (27600 M.) eingestellt; zur Beschaffung von Apparaten etc. für dasselbe $6000 \mathrm{M}$. Für die Augenklinik soll eine Trachombaracke (75 $000 \mathrm{M}$.) errichtet werden. Bei einzelnen Kliniken sind Fehlbeträge (ca. 26000 M.) zu decken. - In Berlin sind für den Unterricht in der Hydrotherapie $29500 \mathrm{M}$. ausgeworfen; dafür sollen im neuen Maschinenhause der Charité die erforderlichen Einrichtungen geschaffen werden. Zur Beschaffung von Mikroskopen etc. für das Anatomische Institut sind 5000 M. eingestellt. Bei der medicinischen Poliklinik und dem physiologischen Institut sind Fehlbeträge zu decken, einige Kliniken und sonstige Institute erfordern bauliche Instandsetzungen. Zur Beschaffung von Apparaten etc. für die Nervenklinik (Projectionsapparat, Apparate für Uebungstherapie und Medicomechanik etc.) sind $18000 \mathrm{M}$. ausgeworfen. Zur Einrichtung des Instituts für Röntgen-Untersuchungen, 
das sich „immer mehr zu einem unentbehrlichen Hilfsmittel für die in der Charité befindlichen Kliniken entwickelt hat", soll ein der Charité benachbartes Haus angekauft und mit einigen Räumen seines Erdgeschosses verwandt werden; für den Ausbau und die innere Einrichtıng der für das Institut bestimmten Rüumme sind ca. $13000 \mathrm{M}$. bestimmt. Für die Charité sind von dem durch das Gesetz aus dem Jahre 1897 eröffneten Credit von 16 Millionen Mark 967300 M. in Anspruch zu nehmen. - Für die Universität Greifswald beansprucht das anatomische Institut zur Ausstattung des Erweiterungsbaues mit Instrumenten etc. 7000 M., zll ballichen Veränderungen 5000 M. Fïıdie chirurgische Klinik sollen für $2000 \mathrm{M}$. Instrumente und Apparate beschafft werden. Zum Neubau der chilurgischen Klinik ist die erste Rate mit $120000 \mathrm{M}$. eingestellt (die Gesammtkosten sind anf $553000 \mathrm{M}$. veranschlagt). Die Frauenklinik erfordert einen Un- und Erweiterungsbau, für den 120000 M. als erste Rate (Gesamintkosten 172200 M.) bewilligt sind. Das hygienische Institut soll Apparate und Instrumente für 6000 M. erhalten. - Für den Neubau des anatomischen Instituts in Breslau wird eine Ergänzungsrate von $10000 \mathrm{M}$. gefordert. Bei den klinischen Anstalten ist ein Fehlbetrag von $10000 \mathrm{M}$. zu decken. Eine Kinderklinik und -Poliklinik soll mit einem Aufwande von 118500 M. gebaut werden; als erste Rate sind 100000 M. eingestellt. Das hygienische Institut soll Apparate etc. für $5000 \mathrm{M}$. erhalten. - In Halle bedarf die psychiatrische und Nervenklinik baulicher Erweiterungen, bezw. Veränderungen (30512 M.). Zur Erweiterung der medicinischen Klinik sind $97400 \mathrm{M}$. eingestellt. Die Begriindung dieser Rate ist in der That mehr als ausreichend. Bisher waren nümlich die geschlechtskranken Prostitıirten, welche die medicinische Klinik aufzumehmen hat, in einem Kellergeschoss der Klinik untergebracht, das nicht nur nach dem Urtheil der Baupolizei zur Unterbringung von Kranken ungeeignet ist, sondern sogar regelmässio. stark mit Kranken überfüllt war. Ausserdem aber „,besteht eine unausgesetzte Verbindıng zwischen den kranken Prostituirten und ilıren Zuhältern. Die letzteren dringen Nachts in das klinische Gebüude ein, erbrechen die Eisenstangen, mit denen die Fenster der Rüume ver-gittert sind, und suchen die Mädchen ins Freie zll bringen und mitzunehmen"(!). Wie lange diese erbanlichen Verhältnisse bestehen und ob wirklich seitens des Leiters der Klinik niemals vorher ihre Aenderung beantragt worden ist, darüber sagt der Bericht nichts ans. - Für das pliarmakologische Institut sind 6000 M. zu Instrumenten etc. erforderlich, für das hygienische Institut 7500 M. zu Apparaten etc. In $\mathrm{Kiel}$ sind zur Erweiterung der Frauenklinils als "Ergänzungsrate“ 12000 M. eingestellt. Zum Neubau der medicinischen Klinik, der in der letzten Zeit bekanntlich Gegenstand lebhafter Erörterungren war, ist die dritte Rate (120000 M.) eingestellt. Nach unseren Informationen ist neuerdings begründete Aussicht vorhanden, dass Geheimrath Qu in $\mathrm{ck}$ e auch der neuerbauten Klinik als Leiter erhalten bleibt. Zum Neuban einer Universitäts-Irrenklinik ist die zweite Rate $(250000 \mathrm{M}$.) ausgeworfen. Das hygienische Institut beansprucht $5000 \mathrm{M}$. für weitere Arbeitsplätze und Mikroskope. Zur Ausführung baulicher Verändel'ungen der chirurgischen Klinik sind 9500 M. nöthig. - In G öt tingen sind zum Um- und E1-weiterungsbau des anatomischen Instituts $5750 \mathrm{M}$., zur Ausstattung des hygienischen Instituts 5000 M., zur Erweiterung der Poliklinik für Ohrenkrankheiten 8000 M., zur Deckung eines Fehlbetrages der klinischen Anstalten 18800 M. eingestellt. (Mit grossem Interesse werden die Göttinger Studenten vernehmen, dass der dortige Karzer umgebaut werden soll; hoffentlich wird dabei den verfeinerten Bedürfnissen der Nenzeit Rechnung getragen). - In M a rbul ro ist zum Nenbau des anatomischen Instituts die zweite Rate eingestellt (150000 M.). Zur Anstellung von Versuchen mit Tuberkulosegiftpräparaten gegen die Rindertuberkulose sind 9000 M. erforderlich. Die chirurgische Klinik hat einen Fehlbetrag von $12500 \mathrm{M}$. Das pharmaceutisch-chemische Institut beanspruclit zur Erweiterung und inneren Ausstattung 52500 M. - In Bonn werden für bauliche Arbeiten mehrerer medicinischer Institute ca. 120000 M. benöthigt. Die Klinik für Hantkrankheiten soll neue Apparate etc. für 8000 M. erhalten. Zum Neuban der Augenklinik ist die erste Rate mit 120000 M. eingestellt. (Gesammte Baukosten 254000 M.).

Schliesslich ist zu bemerken, dass die Absicht besteht, künftig von drei zu drei Jahren eine Statistik der preussischen Universitäten, einschliesslich unter anderem der Kaiser-Wilhelms-Akademie, durch das statistische Bureau anfertigen zu lussen, die über die Zahl, Staatsangehörigkeit und Heimath der Studirenden, über den Lehrkörper, die Vorlesungen und die Kosten des Betriebes in erschöpfender Weise Auskunft geben soll. Die Kosten sind auf $5400 \mathrm{M}$. veranschlagt; die Summe ist zum ersten Male dieses Jahr eingestellt.

In dem Abschnitt Medicinalwesen werden für die Charité zur Deckung eines Fehlbetrages bei den sächlichen Ausgabefonds 1100000 M. gefordert. Der Fehlbetrag ist seit dem Rechnungsjahr 1894/95 zum veitaus grössten Theile durch Einnahmeausfälle, und zwar in Fologe der räımlich nothwendigen Verminderung der Bettenzahl entstanden. Merkwiirdig ist allerdings, dass - nach der Angabe des Etats - erst bei den Verhandlungen über den Neubau der Charité diese Thatsachen, welche sich nach und nach in Folge des Krankenandlanges herausgebildet haben, voll bekannt geworden sind. Zur „Herstellung geordneter. finanzieller Zustände" soll der oben genannte einmalige Zuschuss und die dauernde Erhöhung der Curkosten auf 2,50 M. für Erwachsene und 2,00 M. für Kinder dienen. - Zulr Ergänzung des hygienischen Instituts in Posen ist ein Betrag von $4500 \mathrm{M}$. erforderlich. - Zur Bekämpfung der Granulose werden wie im Vorjahre 350000 M. gefordert, „zumal durch eingehende Untersuchungen sich ein ausgedehnteres und gehäıfteres Torkommen von Granulose auch in anderen, als den bisher bekannten Bezirken herausgestellt hat." - Zur Erforschung der Maull und Klauense uche sind wiederum 30000 M. gefordert. - Zur Einrichtung von psychiatrischen Fortbildungscursen für Regierungsmedicinalräthe ind Kreisphysiker werden wiederum $40000 \mathrm{M}$. verlangt. 